\title{
BIOMECHANICAL ANALYSIS OF NONCONSTRAINED AND SEMICONSTRAINED TOTAL ELBOW REPLACEMENTS: A PRELIMINARY REPORT
}

\author{
K.-S. Shih * \\ Institute of Biomedical Engineering \\ National Taiwan University \\ Taipei, Taiwan 10617, R.O.C. \\ Orthopaedic Division of Surgical Department \\ Far Eastern Memorial Hospital \\ Taipei, Taiwan 22060, R.O.C. \\ T.-W. Lu ${ }^{* *}$ Y.-C. Fu * \\ S.-M. Hou ${ }^{* * *}$ \\ Institute of Biomedical Engineering \\ National Taiwan University \\ Taipei, Taiwan 10617, R.O.C. \\ Department of Orthopaedic Surgery \\ National Taiwan University Hospital \\ Taipei, Taiwan 10051, R.O.C. \\ J.-S. Sun ${ }^{* * *}$ \\ Department of Orthopaedic Surgery \\ Taipei City United Hospital \\ Taipei, Taiwan 10341, R.O.C.

\section{C.-Y. Cheng "} \\ Department of Orthopaedic Surgery \\ Chang Gung Memorial Hospital \\ Taoyuan, Taiwan 33378, R.O.C.
}

\begin{abstract}
The development of elbow arthroplasties has significantly improved the quality of life for many patients suffering from disabling elbow disorders. However, the high complication rate such as loosening and instability limits the long term use of total elbow replacement (TER). In the present study, biomechanical analyses on patients with unilateral nonconstrained (Souter-Strathclyde) and semiconstrained (Coonard-Morrey) TER subjects were performed to investigate differences of their motion patterns under unloaded and loaded conditions. In a biomechanical laboratory, each subject performed vertical and horizontal elbow flexion/extension first without and then with external loading $(5 \mathrm{lb})$. The kinematic data were measured using 3D motion analysis system and the motion axis of the elbow was calculated by a well-defined mathematic model. During these tests, the upperarm was fixed with a special fixation device and their forearms fully supinated. The elbow motion patterns of the affected sides were compared with those of the normal sides and between different conditions.

The results revealed that the elbows moved about a relatively fixed axis both in the semiconstrained and nonconstrained groups in the vertical flexion/extension with or without external loading, compatible with the normal elbows. However, the nonconstrained elbows were less stable during horizontal flexion/externsion motion with or without external loading and the elbow axis moved significantly, indicating of less stability. We conclude that this methodology of elbow motion analysis is acceptable and can be widely recommended for total elbow study. Moreover, the nonconstrained TER is less stable than the semiconstrained TER during the horizontal movement, which was compatible with previous clinical results. The patients with nonconstrained TER are suggested to avoid using their elbows in horizontal motion, especially in loaded conditions.
\end{abstract}

Keywords : Screw axis, Elbow joint, Total elbow arthroplasty, Kinematics.

\section{INTRODUCTION}

Total elbow replacement (TER) has been an established procedure to treat patients with rheumatoid arthritis, posttraumatic arthritis, osteoarthritis and failed reconstructed elbows in the last decade [1,2]. However, the high complication rate such as loosening, polyethylene wear, infection, dislocation and instability has limited the long term survivorship of TER. Moreover, the revision of failed TER can be very chal-

* M.D. ** Professor, corresponding author *** Professor 
lenging compared to revisions of total knee and hip replacements owing to limited metaphyseal bone stock and soft tissue envelope [3-6].

Movement of the elbow joint is controlled by a complex interaction of the articular surfaces, ligaments and surrounding muscles. The normal kinematics of the elbow has been studied thoroughly in the past and it is generally accepted that the elbow joint represents the most hinge-like joint in all of the human joints during flexion/extension, with a slight carrying abduction angle [7-11]. There are three types of designs of TER, namely constrained, semiconstrained and nonconstrained, all aiming to recover the full function of the diseased or injured elbow. Constrained TERs use simple hinged joints, the intrinsic articulation constraints of which predictably resulted in failure by loosening [1]. The constrained TER has no longer been used because of this reason and semiconstrained and nonconstrained types became the current trend for total elbow arthroplasty. However, controversies exist in the literature as to which type of TER should be selected for elbow arthroplasty [3,5,11-15]. This is because there has no objective evidence showing the capacity of either type in reconstructing completely the elbow movement function. Moreover, the mechanism of the failure in these TERs has not been well understood, increasing the uncertainty in the selection process. The unconstrained design depends on the geometry of the prosthesis, surrounding capsuloligamentous structures, and static and dynamic muscular contributions to stability [16]. The main concern of this type is the development of instability which is attributable to numerous factors including prosthesis design, ligament integrity and the insert position of the prosthesis [1]. Semiconstrained TER has less dependence on soft tissue stability and thereby the indication has been expanded to include ligament attenuation and bone loss [16]. However, these have not prevented semiconstrained prostheses from instability, polyethylene wear and loosening $[2,17,18]$. Moreover, the initial massive bony resection for semiconstrained TER makes the revision operation rather difficult. Biomechanical factors related to TER designs determine the failure mechanism of TER and are thus critical to the survivorship. At present, knowledge of the interactions between the force bearing joint surface and periarticular structures of the TER comes mainly from in vitro studies $[1,14,15,19,20]$ because in vivo measurements of the forces transmitted in these structures are difficult [3]. While difficult to measure these force interactions directly, their effects on the total joint components, which are the resulting kinematics, can be measured given appropriate techniques. Biomechanical analysis of TERs through the measurement of their in vivo kinematics can provide critical information for the improvement of future design and implantation of TERs.

Measurement of the elbow motion in vivo in the literature has been through the use of electromagnetic tracking devices $[9,10,14,15]$ and roentgen stereophotogrammetry analysis (RSA) [3,11]. The accuracy of the electromagnetic tracking devices can be affected by metals nearby, limiting its application on patients with metallic implants. RSA is of high accuracy but it is limited to static measurements. An alternative for noninvasive, in vivo 3D measurement of joint kinematics is the use of video stereophotogrammetry which has been widely used in gait analysis [21-24]. With the method the body segment motion is calculated from trajectories of skin markers reconstructed from images captured by cameras. A main source of errors in this method is the errors due to the relative movement between the skin markers and the underlying bone, called skin movement artifacts. The application of the method in the measurement of elbow kinematics has to take account of the skin movement artifacts.

There are several methods that can be used to describe the kinematics of the elbow joint. Among the existing methods, screw axis representation is regarded as a precise and sequence independent method for the description of the elbow joint kinematics in three- dimensions (3D) $[9,10]$. With this representation, the screw axis will remain fixed relative to the humerus and ulna during elbow flexion/extension if the elbow joint is a hinge joint with a unique axis of motion. The elbow joint has been regarded by many as a hinge joint but its mobility and stability have been shown to be affected by the type of external loads and force-bearing positions during daily activities [25]. For example, an external load acting perpendicular to the flexion/extension axis of the elbow joint may have significantly different effects on the stability of the joint during flexion/extension movement compared to that acting along the flexion/extension axis. These two types of loading may have very different effects on constrained and nonconstrained TER. No study has ever compared the mobility and stability between the two types of TER during flexion/extension while the forearm is subjected to the two different types of external loads.

The purpose of the present study was to investigate the 3D kinematics of nonconstrained (Souter-Strathclyde) and semiconstrained (Coonard-Morrey) TER during elbow flexion/extension in the vertical and horizontal planes, under loaded and non-loaded conditions. Clinical implications were then discussed in the light of the results.

\section{MATERIALS AND METHODS}

Six subjects (age: $68.3 \pm 10.65 ; 4$ females and 2 males) with unilateral TER, four semiconstrained (Coonard-Morrey) and two nonconstrained (SouterStrathclyde), were recruited from National Taiwan University Hospital and Chang Gung Memorial Hospital. All of them had good postoperative results with nearly full and free range of motion of the elbow joint. Theses two total elbow types were the only products that were used in the last decade in Taiwan. The purpose and procedures of the study were explained to the subjects and informed consent forms were obtained prior to the experiments. The contralateral elbows of 
the same subjects were used as controls. The radiographs of the semiconstrained and nonconstrained TER's from two typical subjects are given in Fig. 1.

The subjects performed elbow flexion/extension in a gait laboratory equipped with a 7-camera motion analysis system (Vicon 512, Oxford Metrics Ltd., U.K.) operating at a frequency of $60 \mathrm{~Hz}$. Light-weight retroreflective markers were placed on the ulna styloid process, radial styloid process, olecranon, and the middle of the ulna to track the motion of the forearm. The subject was seated with the trunk stabilized in the chair and the upper arm supported and fixed by a stainless fixator attached to the table by two C-shaped clips, Fig. 2. The fixator was adjusted to fit the upper arm to ensure the upper arm is stable and stationary. Eight retroreflective markers were also attached on the fixator to define its position and thus that of the upper arm. For each subject, both of the affected (replaced) and unaffected elbows were tested. The subject performed elbow flexion/extension in the vertical and horizontal planes without holding a weight and when holding a dumbbell of $1 \mathrm{~kg}$ while marker trajectories were recorded by the motion capture system. The measured marker trajectories were used to define and describe the motions of the coordinate systems for the forearm and upper arm from which the screw axis of the elbow joint was then calculated.

To calculate the motion of the screw axis, or helical axis, rotation matrices and translation vectors of the two rigid bodies, upper arm and forearm, were needed. The screw axis method describes the general motion of a rigid body by the translation along and rotation about an imaginative axis. The method is free from the sequence dependence and gimble lock problems associated with representations using Euler and/or Cardan angles. However, screw axis can be very sensitive to movement noises such as skin movement artifacts. The screw axis method proposed by Spoor and Veldpaus [26] and further developed by Woltring et al. [27] was the first which considered skin movement artifacts when describing the general motion of a rigid body. In the present study, the screw axis calculation was based on their methods.

The movement of a rigid body from position 1 to position 2 can be described by a translation vector $\vec{v}$ and a rotation matrix $R$ that satisfies

$$
R^{T} R=I
$$

where $I$ is the $3 \times 3$ unit matrix and the superscript $\mathrm{T}$ indicates transposition. Let $\vec{a}_{1}, \vec{a}_{2}, \ldots, \vec{a}_{n}$ denote the radius vectors of $\mathrm{n}(\mathrm{n} \geqq 3)$ non-collinear points $\mathrm{A}_{1}$, $\mathrm{A}_{2}, \ldots, \mathrm{A}_{\mathrm{n}}$ of the body in position 1 , then the radius vectors $\vec{q}_{1}, \vec{q}_{2}, \ldots, \vec{q}_{n}$ of these points in position 2 are given by

$$
\vec{q}_{i}=R \vec{a}_{i}+\vec{v} \quad \text { for } \quad i=1,2, \ldots, \mathrm{n} .
$$

$\mathrm{R}$ and $\mathrm{v}$ are unknown and must be determined from the measured radius vectors $\vec{p}_{1}, \vec{p}_{2}, \ldots, \vec{p}_{n}$ of $\mathrm{A}_{1}, \mathrm{~A}_{2}, \ldots$, $A_{n}$ in position 2, which in general will differ from the

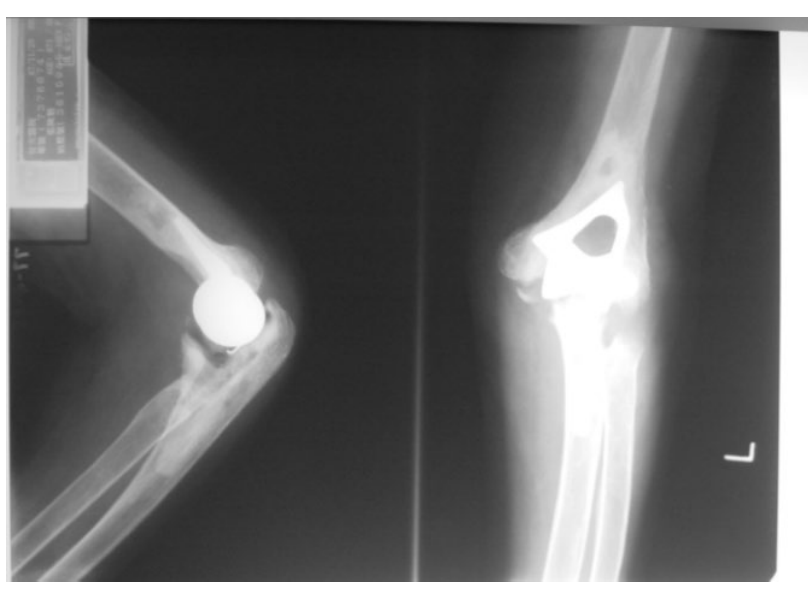

(a)

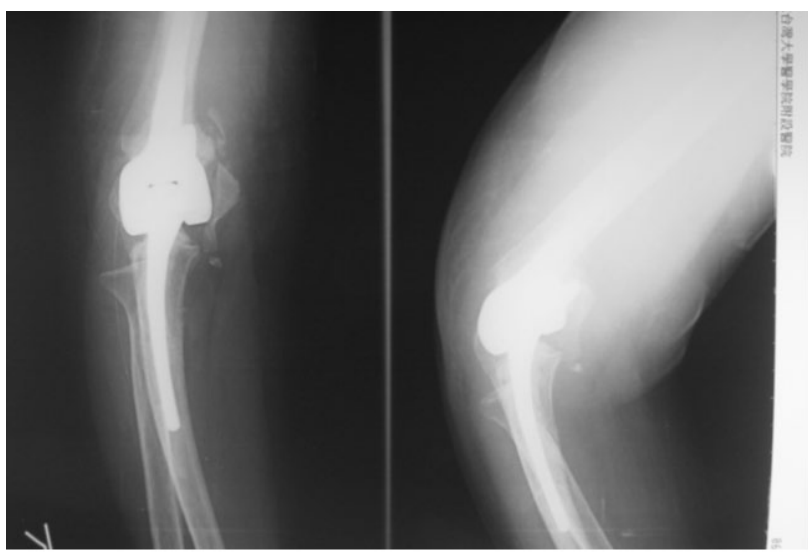

(b)

Fig. 1 Radiographs of (a) nonconstrained and (b) semiconstrained TER's of two typical subjects

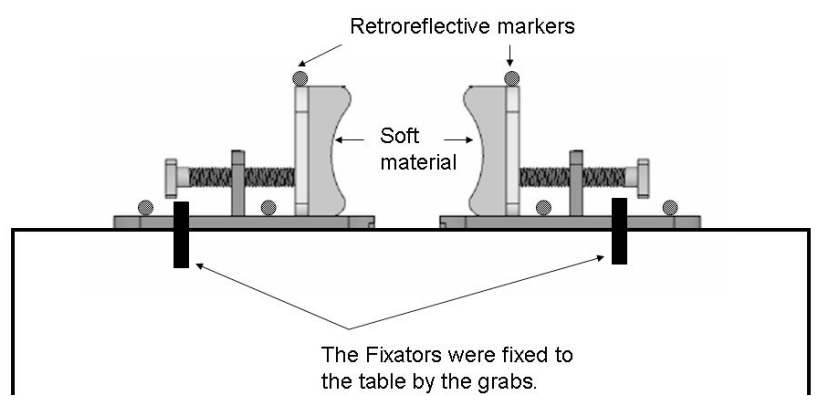

Fig. 2 The upper arm fixator was designed to fix the humerus during motion on the table. Markers attached on it were used to define local coordinate system of the humerus

exact vectors $\vec{q}_{1}, \vec{q}_{2}, \ldots, \vec{q}_{n}$. Generally, $\vec{v}$ and $R$ can be determined by minimizing the mean squared errors $f(\vec{v}, R)$ defined as

$$
f(\vec{v}, R)=\frac{1}{n} \sum_{i=1}^{n}\left(R \vec{a}_{i}+\vec{v}-\vec{p}_{i}\right)^{T}\left(R \vec{a}_{i}+\vec{v}-\vec{p}_{i}\right)
$$

Given the $\vec{v}$ and $R$, the screw axis parameters of this 
motion can then be determined. With the screw axis method, the general motion of a rigid body is represented by a translation $t$ and rotation $\theta$ along an axis in 3D. The rotation angle $\theta$ is defined by the righthanded rule, and will always be nonnegative and equal to or less than $\pi$, Fig. 3. For a point $P$ on the rigid body with its local position vector $\vec{a}$, global position vector $\vec{P}_{1}$ at position 1 and $\vec{P}_{2}$ at position $2, \vec{P}_{2}$ is usually represented as

$$
\vec{P}_{2}=R \vec{a}+\vec{v}
$$

Another way to describe the relationship between $\mathrm{P}_{1}$ and $\mathrm{P}_{2}$ is that $\mathrm{P}_{1}$ first moves along an axis to $\mathrm{P}_{1}{ }_{1}$ and then rotates to $\mathrm{P}_{2}$ (Fig. 3). If the axis is a unit vector $\vec{u}$ and the radius vector $\vec{r}$ is perpendicular to the axis, then

$$
\vec{u} \cdot \vec{u}=1 \quad \text { and } \quad \vec{u} \cdot \vec{r}=0
$$

The movement of the rigid body defined in Eq. (4) can be described alternatively as:

$$
\begin{aligned}
\vec{P}_{2}= & \vec{a}+t \vec{u}+(1-\cos \theta) \vec{u} \times[\vec{u} \times(\vec{a}-\vec{r})] \\
& +(\sin \theta) \vec{u} \times(\vec{a}-\vec{r})
\end{aligned}
$$

Comparison between Eqs. (4) and (6) gives

$$
\vec{v}=t \vec{u}+(1-\cos \theta) \vec{r}-\sin \theta(\vec{u} \times \vec{r})
$$

and

$$
R \vec{a}=\vec{a} \cos \theta+(1-\cos \theta) \vec{u}(\vec{u} \cdot \vec{a})+\sin \theta(\vec{u} \times \vec{a})
$$

From Eq. (8), we further have

$$
\frac{1}{2}\left(R-R^{T}\right) \vec{a}=\sin \theta(\vec{u} \times \vec{a})
$$

and $\frac{1}{2}\left(R+R^{T}\right)=\cos \theta I+(1-\cos \theta) \vec{u} \vec{u}^{T}$

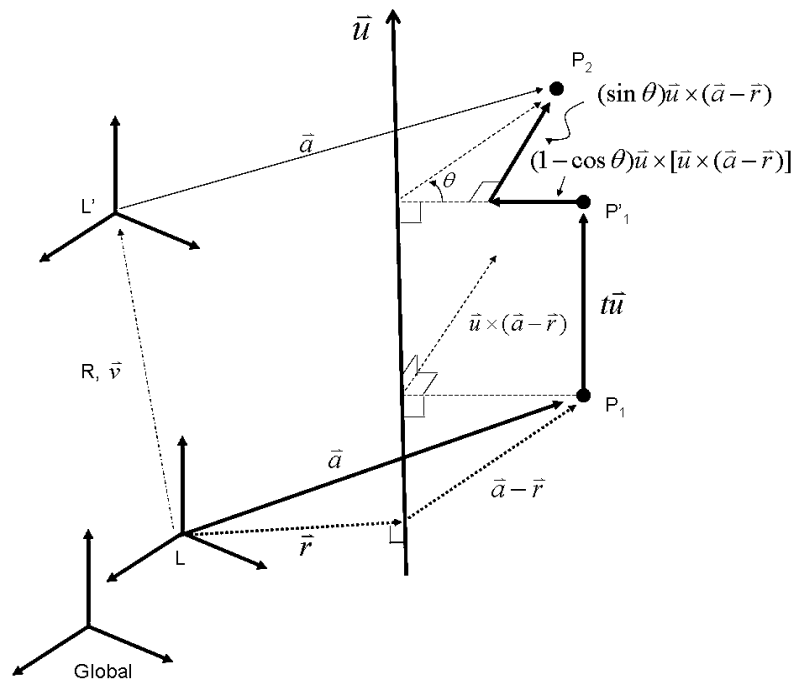

Fig. 3 The determination of the screw axis of the elbow joint during motion
Since the matrix $\frac{1}{2}\left(R-R^{T}\right)$ is an oblique- symmetric matrix, $\vec{u} \sin \theta$ is given by:

$$
\vec{u} \sin \theta=\frac{1}{2}\left[\begin{array}{l}
R_{32}-R_{23} \\
R_{13}-R_{31} \\
R_{21}-R_{12}
\end{array}\right]
$$

where $R_{\mathrm{xy}}$ is the component of the rotation matrix $R$.

For $\sin \theta \geq 0$ and considering Eq. (5), Eq. (8) can be solved as:

$$
\sin \theta=\frac{1}{2} \sqrt{\left(R_{32}-R_{23}\right)^{2}+\left(R_{13}-R_{31}\right)^{2}+\left(R_{21}-R_{12}\right)^{2}}
$$

On the other hand, $\cos \theta$ can be calculated from Eq. (7) by adding up the principal diagonal terms, which results in:

$$
3 \cos \theta+(1-\cos \theta) \operatorname{trace}\left(\vec{u}^{T}\right)=\operatorname{trace}\left(\frac{1}{2}\left(R+R^{T}\right)\right)
$$

where trace $(\mathrm{M})$ means the sum of the principal diagonal terms of the matrix M. Since $\operatorname{trace}\left(\vec{u} \vec{u}^{T}\right)=\vec{u}^{T} \vec{u}=1$, it follows:

$$
\cos \theta=\frac{1}{2}\left(R_{11}+R_{22}+R_{33}-1\right)
$$

For numerical reasons, it is suggested Eq. (9) be used to solve $\theta$ if $\sin \theta \leq 0.5$ and Eq. (11) otherwise [26]. The unit vector $\vec{u}$ can be determined once $\sin \theta$ is known from Eq. (9), and the translation $t$ and radius vector $\vec{r}$ can be determined from Eqs. (5) and (7). Detailed descriptions of the above derivations can also be found in Spoor and Veldpaus [26].

In the present study, the differences of the unit vector of the screw axis between two TER types are compared during flexion and extension motion. Standard deviations of the unit vectors of the screw axes were calculated to evaluate the differences.

\section{RESULTS}

The motion of the screw axis of the semiconstrained TER during a typical trial is given in Fig. 4. The screw axis moved more during horizontal flexion/extension than during vertical flexion/extension either with or without loading. On the other hand, the motion of the screw axis seemed more stable under loaded condition in both horizontal and vertical motions. The results also revealed that the motion of the screw axis seemed more stable while performing flexion motion than extention, especially in loaded condition. Generally, the screw axis of the semiconstrained TER was closer to a fixed axis in the vertical flexion/extension with or without external loading. For the nonconstrained TER, the screw axis pattern of a typical trial is shown in Fig. 5. The screw axis motions moved more for 


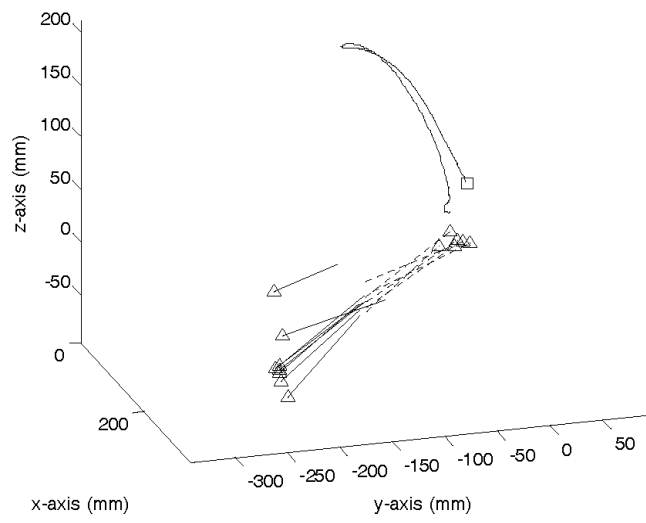

(a)

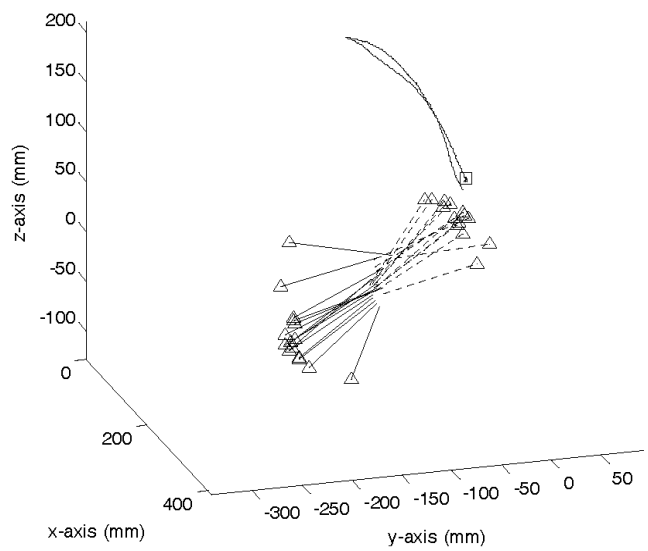

(b)

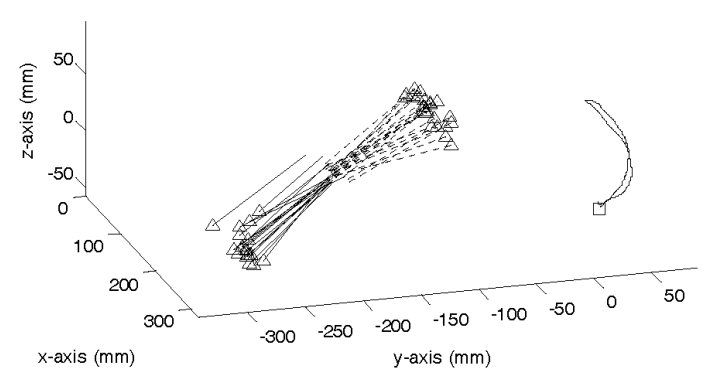

(c)

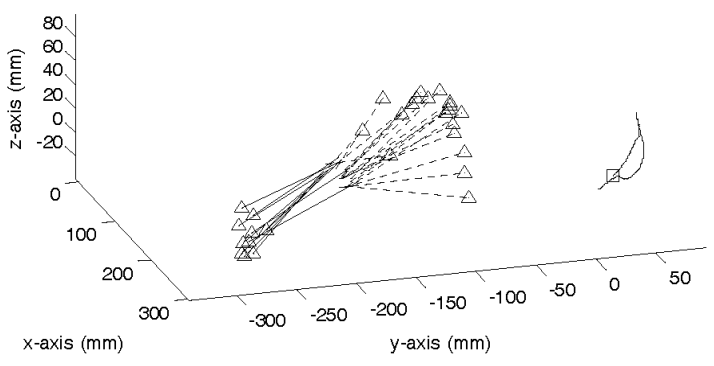

(d)

Fig. 4 Variations of the screw axis during flexion-extension of (a) vertical, (b) vertical with dumbbell, (c) horizontal, and (d) horizontal with dumbbell of the semiconstrained TER of a typical trial. The curve with a square marker is the trajectory of the ulna marker, which represents the motion of the elbow. Lines with triangle markers were the screw axes during motion: solid lines during flexion and dashed lines during extension

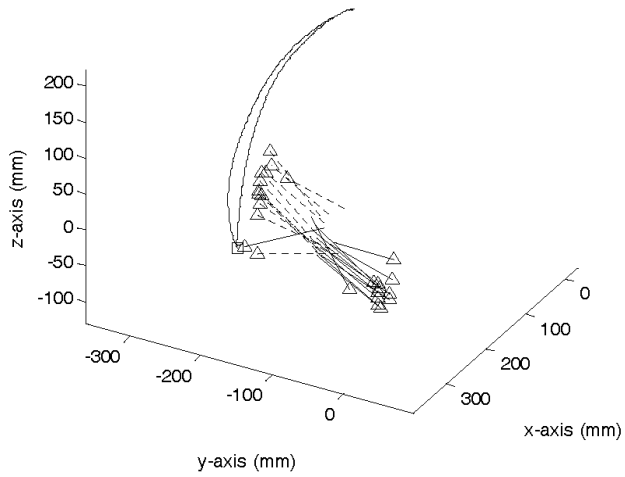

(a)

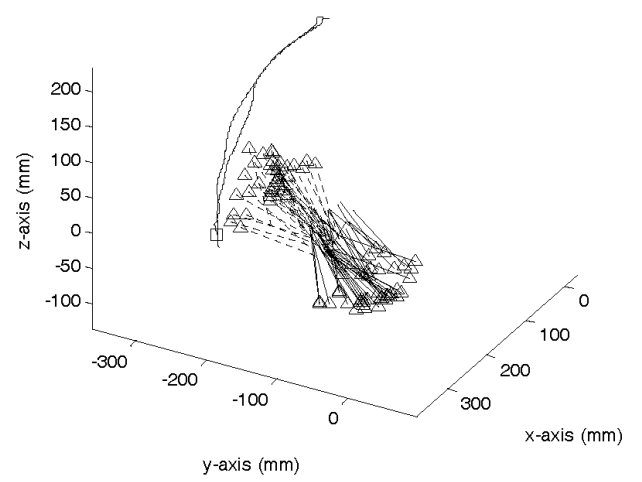

(b)

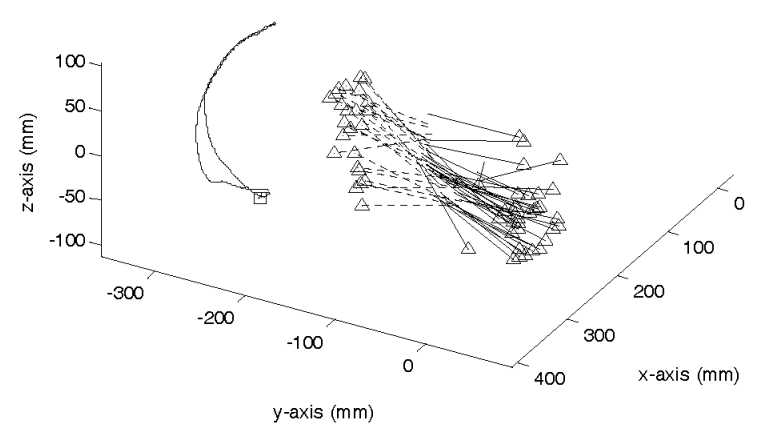

(c)

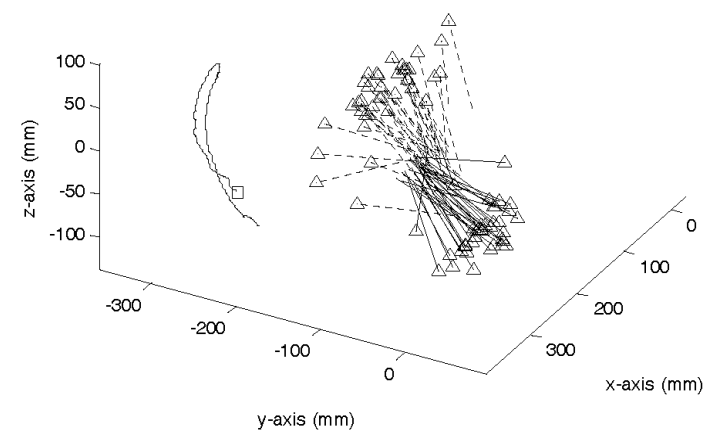

(d)

Fig. 5 Variations of the Screw axis during flexionextension of (a) vertical, (b) vertical with dumbbell, (c) horizontal, and (d) horizontal with dumbbell of nonconstrained TER of a typical trial. The curve with a square marker is the trajectory of the ulna marker, which represents the motion of the elbow. Lines with triangle markers were the screw axes during motion: solid lines during flexion and dashed lines during extension 
nonconstrained TER than for semiconstrained TER in both motion planes and loading conditions. The effects of motion plane on the motion of the screw axis in nonconstrained TER were similar to those in the semiconstrained TER. However, in contrast to the semiconstrained TER's, the screw axis was less stable during loaded motion.

The means and standard deviations of the positions of the screw axis during flexion and extension are listed in Tables 1 and 2, respectively. The results showed that the ranges of motion of the screw axis of the semiconstrained and nonconstrained TER were similar in vertical motion. However, they were different in horizontal motion, especially under loaded conditions.

Table 1 The means and standard deviations of the screw axes of the semiconstrained and nonconstrained TER during elbow flexion.

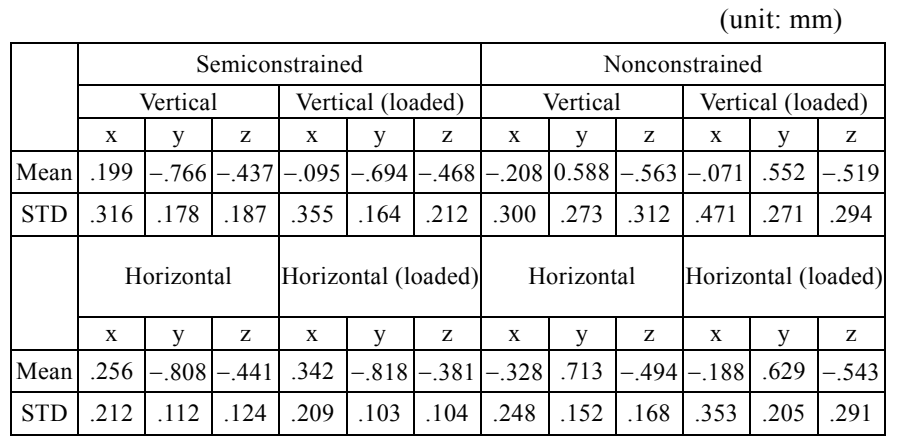

Table 2 The means and standard deviations of the screw axes of the semiconstrained and nonconstrained TER during elbow extension.

(unit: $\mathrm{mm}$ )

\begin{tabular}{|c|c|c|c|c|c|c|c|c|c|c|c|c|}
\hline & \multicolumn{6}{|c|}{ Semiconstrained TER } & \multicolumn{6}{|c|}{ Nonmiconstrained TER } \\
\hline & \multicolumn{3}{|c|}{ Vertical } & \multicolumn{3}{|c|}{ Vertical (load) } & \multicolumn{3}{|c|}{ Vertical } & \multicolumn{3}{|c|}{ Vertical (load) } \\
\hline & $x$ & $\mathrm{y}$ & $\mathrm{z}$ & $\mathrm{x}$ & $\mathrm{y}$ & $\mathrm{z}$ & $\mathrm{x}$ & $\mathrm{y}$ & $\mathrm{z}$ & $\mathrm{x}$ & $\mathrm{y}$ & $\mathrm{z}$ \\
\hline Mean & -.296 & .804 & .316 & -.111 & .666 & .559 & .195 & -.628 & .644 & .097 & -0.515 & .605 \\
\hline \multirow[t]{3}{*}{ STD } & .209 & .186 & .172 & .288 & .139 & .196 & .197 & .0191 & .236 & .424 & .268 & .264 \\
\hline & \multicolumn{3}{|c|}{ Horizontal } & \multicolumn{3}{|c|}{ Horizontal (load) } & \multicolumn{3}{|c|}{ Horizontal } & \multicolumn{3}{|c|}{ Horizontal (load) } \\
\hline & $\mathrm{x}$ & $\mathrm{y}$ & $\mathrm{z}$ & $\mathrm{x}$ & $\mathrm{y}$ & $\mathrm{z}$ & $\mathrm{x}$ & $\mathrm{y}$ & $\mathrm{z}$ & $\mathrm{x}$ & $\mathrm{y}$ & $\mathrm{z}$ \\
\hline Mean & -.120 & .873 & .345 & -.196 & .798 & .369 & .262 & .772 & .437 & .169 & -.542 & .601 \\
\hline STD & .211 & .086 & .198 & .353 & .141 & .190 & .257 & .131 & .226 & .302 & .301 & .338 \\
\hline
\end{tabular}

\section{DISCUSSION}

The dynamic stabilization mechanism of a replaced joint is undoubtedly important for its clinical performance and likely will have an effect on the functional long-term survival of the prosthesis [1]. However, almost all the biomechanical studies of total elbow replacements were based on cadarveric specimens and testing conditions simulating muscle loadings that were very different from in vivo conditions. In vitro studies have helped establish important knowledge for TER kinematics, but contributions of soft tissue restrictions and muscle stabilization function were not considered. In vivo tests are not subject to the above-mentioned limitations but measurements have to be made noninvasively. In the current study the experimental setup and the three-dimensional motion capture system enabled a thorough quantitative rigid body movement assessment of the complex kinematics of semiconstrained and nonconstrained total elbow replacements. The motion of the elbow joint was represented as the rigid body motion of the forearm relative to the humerus using screw axis method while considering skin movement artefacts. The results in this preliminary study agreed well with clinical observations, suggesting that the experimental and theoretical methods proposed in the present study can be used to evaluate the kinematic performance of different designs of TER.

The results of the current study showed that nonconstrained TER was less stable than semiconstrained TER (Figs. 4 and 5, Tables 1 and 2), in agreement with both clinical observations and previous cadaveric studies such as An [1] who compared the kinematics of Morrey type and nonconstrained type TER. In the present study elbow flexion/extension motions were studied not only in the vertical plane considered in most previous studies but also in the horizontal plane. Motions in both planes represent a range which covers most situations one would face in most daily activities. The horizontal plane motion subjected the elbow to external varus loadings. In An's in vitro study a varus stress was also applied to the TER. However, in vivo motions are a result of dynamic muscle activities and ligamentous constraints so the actual loading transmitted by the TER may be very different from a single unidirectional varus stress that was simulated in vitro. In the current study the nonconstrained TER was found to be stable in the vertical motion and less so during horiziontal motion as shown by the standard deviations of the screw axes (Tables 1 and 2), which has not been found in previous in vitro studies using a simulated stress. This finding suggests that avoiding horizontal motion of nonconstrained TER may be necessary to reduce its instability.

Although semiconstrained TER was found to be more stable during elbow flexion/extension in both the vertical and horiziontal planes compared to nonconstrained TER, it requires more bone resections at surgery and may have complications such as loosening, infection, osteolysis and wear. Moreover, revision of semiconstrained TER is difficult because of the weak and reduced bone stalk at the elbow after semiconstrained total elbow arthroplasty. Nonconstrained TER was found to be less stable than semiconstrained type but its clinical results have been good with good survivorship and less bone resection during surgery. During a revision total elbow arthroplasty, a nonconstrained TER is often suggested. Therefore, if the bone stalk and collateral ligaments are good, nonconstrained implants remain an option for inflammatory arthritis and good long-term results have been reported [16]. 
The screw axis motions during vertical motion without external loading in the present study were similar to those in previous studies of normal elbow joint $[9,10]$. No such data for TER were reported in the literature. Both Bottlang et al. [9] and Duck et al. [10] used cadaveric specimens to study screw axis motion of normal elbow joint under simulated muscle forces. The results of the current study were similar to theirs but with higher variances, most likely because of the fundamental differences between TER and normal cadaveric joints. As pointed out above, the forces generated by muscles in vivo were difficult to simulate in cadaveric specimens. The articular surfaces and ligaments, which would greatly influence the performance of the joint during motion, were also different between TER and normal cadaver joints. In the present study, the articular surfaces were determined by the design of the TER, which can be very different from those of the normal elbow joint. Some main ligaments were also released during total elbow arthroplasty. With these differences it was inevitable that our results had bigger variations than those of the previous studies.

Previous studies on total elbow kinematics considered only those during vertical motion even though the elbow joint during daily activities involved motions in all planes [25]. In the present study elbow flexion/extension motions were studied using screw axis method both in the vertical and horizontal planes to cover most situations one would face in most daily activities. As shown in Figs. 4 and 5 and Tables 1 and 2, the nonconstrained TER was more unstable than semiconstrained TER during horizontal flexion/extension, especially under loading. Several biomechanical factors might contribute to the results, including the position and orientation of the forearm relative to the upper arm, the TER design, remaining ligamentous structures and strength of the surrounding muscles. Under loaded condition, the elbow was subjected to the external moments applied by the weight of forearm and the dumbbell. The stability of the joint depended on the ability of the TER and the surrounding soft tissues in producing the counterbalancing internal moments. The hinge design in semiconstrained TER provided resisting moments when an external moment was applied while external moments at a nonconstrained TER had to be resisted by ligaments and muscles. During vertical motion, external moments were mainly in the sagittal plane so the elbow flexors and extensors were effective in resisting these external loads. During horizontal motion, on the other hand, the external moments were mainly in the transverse and frontal planes. The major elbow muscles were not mechanically effective in resisting these moments so the external moments had to be resisted by the TER structure and the surrounding ligamentous structures. Therefore, the semiconstrained TER was relatively stable even during horizontal motion because of its hinge joint design. On the other hand, since the nonconstrained TER did not have a hinge joint to resist the external moment through the articular surfaces, the stability of the joint depended very much on the strength of the muscles and conditions of the ligamentous structures. This may explain why the elbow joint became more unstable while under a higher load during horizontal motion. The compromised performance of the nonconstrained TER during loaded horizontal motions, when compared to those of the semiconstrained TER, was also consistent with clnical observations.

The results in the current study suggest that the experimental design and the methodology of elbow motion analysis were effective in differentiating performances between different TER designs and can be widely used in future studies on total elbow replacements. The nonconstrained TER was less stable than the semiconstrained TER during loaded horizontal motions. Patients with nonconstrained TER should be cautious in activities involving elbow motions in the horizontal plane especially under loading.

\section{REFERENCES}

1. An, K.-N., "Kinematics and Constraint of Total Elbow Arthroplasty," Journal of Shoulder \& Elbow Surgery, 14, pp. 168S-173S (2005).

2. Lee, B. P., Adams, R. A. and Morrey, B. F., "Polyethylene Wear After Total Elbow Arthroplasty," Journal of Bone \& Joint Surgery, American Volume, 87, pp. 1080-1087 (2005).

3. Valstar, E. R., Garling, E. H. and Rozing, P. M., "Micromotion of the Souter-Strathclyde Total Elbow Prosthesis in Patients With Rheumatoid Arthritis 21 Elbows Followed for 2 Years.," Acta Orthopaedica Scandinavica, 73, pp. 264-272 (2002).

4. Hargreaves, D. and Emery, R., "Total Elbow Replacement in the Treatment of Rheumatoid Disease," Clinical Orthopaedics \& Related Research, 366, pp. 61-71, (1999).

5. O’Driscoll, S. W. and King, G. J., "Treatment of Instability After Total Elbow Arthroplasty," Orthopedic Clinics of North America, 32, pp. 679-695 (2001).

6. Goldberg, V. M., Figgie, H. E., 3rd, Inglis, A. E. and Figgie, M. P., "Total Elbow Arthroplasty," Journal of Bone \& Joint Surgery, American Volume, 70, pp. 778-783 (1988).

7. London, J. T., "Kinematics of the Elbow," Journal of Bone \& Joint Surgery, American Volume, 63, pp. 529-535 (1981).

8. Morrey, B. F. and Chao, E. Y., "Passive Motion of the Elbow Joint," Journal of Bone \& Joint Surgery, American Volume, 58, pp. 501-508 (1976).

9. Bottlang, M., Madey, S. M., Steyers, C. M., Marsh, J. L. and Brown, T. D., "Assessment of Elbow Joint Kinematics in Passive Motion by Electromagnetic Motion Tracking," Journal of Orthopaedic Research, 18, pp. 195-202 (2000).

10. Duck, T. R., Dunning, C. E., Armstrong, A. D., Johnson, J. A. and King, G. J. W., "Application of Screw Displacement Axes to Quantify Elbow Instability," Clinical 
Biomechanics, 18, pp. 303-310 (2003).

11. Ericson, A., Arndt, A., Stark, A., Wretenberg, P. and Lundberg, A., "Variation in the Position and Orientation of the Elbow Flexion Axis," Journal of Bone \& Joint Surgery, British Volume, 85, pp. 538-544 (2003).

12. Giesl, P., Meisel, D., Scheurle, J. and Wagner, H., "Stability Analysis of the Elbow with a Load," Journal of Theoretical Biology, 228, pp. 115-125 (2004).

13. Inagaki, K., O’Driscoll, S. W., Neale, P. G., Uchiyama, E., Morrey, B. F. and An, K.-N., "Importance of a Radial Head Component in Sorbie Unlinked Total Elbow Arthroplasty," Clinical Orthopaedics \& Related Research, 400, pp. 123-131 (2002).

14. O'Driscoll, S. W., An, K. N., Korinek, S. and Morrey, B. F., "Kinematics of Semi-Constrained Total Elbow Arthroplasty," Journal of Bone \& Joint Surgery, British Volume, 74, pp. 297-299 (1992).

15. Schuind, F., O'Driscoll, S., Korinek, S., An, K. N. and Morrey, B. F., "Loose-Hinge Total Elbow Arthroplasty. an Experimental Study of the Effects of Implant Alignment on Three-Dimensional Elbow Kinematics," Journal of Arthroplasty, 10, pp. 670-678 (1995).

16. Ring, D., Kocher, M., Koris, M. and Thornhill, T. S., "Revision of Unstable Capitellocondylar (Unlinked) Total Elbow Replacement," Journal of Bone \& Joint Surgery, American Volume, 87, pp. 1075-1079 (2005).

17. Ramsey, M. L., Adams, R. A. and Morrey, B. F., "Instability of the Elbow Treated with Semiconstrained Total Elbow Arthroplasty," Journal of Bone \& Joint Surgery, American Volume, 81, pp. 38-47 (1999).

18. Wright, T. W. and Hastings, H., "Total Elbow Arthroplasty Failure Due to Overuse, C-Ring Failure, And/Or Bushing Wear," Journal of Shoulder \& Elbow Surgery, 14, pp. 65-72 (2005).

19. King, G. J., Itoi, E., Niebur, G. L., Morrey, B. F. and An, K. N., "Motion and Laxity of the Capitellocondylar Total Elbow Prosthesis," Journal of Bone \& Joint Surgery, American Volume, 76, pp. 1000-1008 (1994).
20. Ramsey, M., Neale, P. G., Morrey, B. F., O’Driscoll, S. W. and An, K.-N., "Kinematics and Functional Characteristics of the Pritchard ERS Unlinked Total Elbow Arthroplasty," Journal of Shoulder \& Elbow Surgery, 12, pp. 385-390 (2003).

21. Gage, J. R., DeLuca, P. A. and Renshaw, T. S., "Gait Analysis: Principle and Applications with Emphasis on its Use in Cerebral Palsy," Instructional Course Lectures, 45, pp. 491-507 (1996).

22. Kadaba, M. P., Ramakrishnan, H. K. and Wootten, M. E., "Measurement of Lower Extremity Kinematics During Level Walking," Journal of Orthopaedic Research, 8, pp. 383-392 (1990).

23. Sutherland, D. H. and Hagy, J. L., "Measurement of Gait Movements from Motion Picture Film," Journal of Bone \& Joint Surgery, American Volume, 54, pp. 787-797 (1972).

24. Lin, H.-C., Lu, T.-W. and Hsu, H.-C, "Comparisons of Joint Kinetics in the Lower Extremity Between Stair Ascent and Descent," Journal of Mechanics, 21, pp. 41-45 (2004).

25. Murray, I. A. and Johnson, G. R., "A Study of the External Forces and Moments at the Shoulder and Elbow While Performing Every Day Tasks," Clinical Biomechanics, 19, pp. 586-594 (2004).

26. Spoor, C. W. and Veldpaus, F. E., "Rigid Body Motion Calculated from Spatial Co-Ordinates of Markers," Journal of Biomechanics, 13, pp. 391-393 (1980).

27. Woltring, H. J., Huiskes, R., de Lange, A. and Veldpaus, F. E., "Finite Centroid and Helical Axis Estimation from Noisy Landmark Measurements in the Study of Human Joint Kinematics," Journal of Biomechanics, 18, pp. 379-389 (1985).

(Manuscript received November 20, 2006, accepted for publication December 29, 2006.) 